\title{
Effects of the Transient Outward Potassium Current on Action Potential Upstroke Velocities Tested using the Dynamic Clamp Technique
}

\author{
Arie O Verkerk, Christiaan C Veerman, Jan G Zegers, Ronald Wilders \\ Academic Medical Center, University of Amsterdam, Amsterdam, The Netherlands
}

\begin{abstract}
The voltage-gated transient outward potassium current ( $\left.I_{\text {tol }}\right)$ plays a prominent role in the early repolarization phase of the cardiac action potential (AP) and thereby contributes to the refractory period and inotropic state of the myocardium. The current is largely responsible for differences in AP repolarization between species, between left and right ventricle, and transmurally, and it is affected by various pathophysiological conditions, such as heart failure.

In voltage clamp experiments, I to1 $_{\text {already activates }}$ during depolarization to potentials near -50 to $-30 \mathrm{mV}$, suggesting that $I_{\text {to1 }}$ may be active during the AP upstroke, but whether it modulates the maximal AP upstroke velocity ( $\left.d V / d t_{\max }\right)$ is unknown. In the present study, we addressed this issue using the dynamic clamp configuration of the patch-clamp technique.

Experimental data were acquired from HEK-293 cells expressing the fast sodium current that is responsible for the rapid cardiac AP upstroke. The fast component of $I_{\text {to }}$ $\left(I_{t o, f}\right)$ was computed in real time and injected into the HEK-293 cell during its upstroke. $I_{\text {to,f }}$ density, activation rate, and voltage dependence were varied.

We conclude that $I_{t o, f}$ may modulate $d V / d t_{\max }$ and $A P$ overshoot, but only if its activation is fast and its activation threshold is near -50 to $-40 \mathrm{mV}$.
\end{abstract}

\section{Introduction}

The transient outward potassium current $\left(I_{\text {to1 }}\right)$ is responsible for the phase- 1 repolarization of the cardiac action potential (AP) and thereby contributes to the refractory period and inotropic state of the myocardium. It has become increasingly clear that $I_{\mathrm{to} 1}$ also modulates conduction velocity through the associated changes in membrane potential $\left(V_{\mathrm{m}}\right)$ during the early action potential plateau phase. However, in voltage clamp experiments, $I_{\text {to1 }}$ already rapidly activates during depolarizing pulses to potentials around -50 to $-30 \mathrm{mV}$ [1]. This suggests that $I_{\text {to1 }}$ may also modulate the maximal AP upstroke velocity
(dV/d $\left.t_{\max }\right)$. However, data on the effects of $I_{\mathrm{to1}}$ on the AP upstroke are lacking.

Presently, various knock-out mouse models exist for $\mathrm{K}_{\mathrm{V}} 4.2$ and $\mathrm{K}_{\mathrm{V}} 4.3$, pore-forming $\alpha$-subunits of the potassium ion channels underlying the fast component of $I_{\mathrm{to1}}\left(I_{\mathrm{to}, \mathrm{f}}\right)[1,2]$. These mouse models clearly demonstrated the effects of $I_{\mathrm{to}, \mathrm{f}}$ on early AP repolarization, but data on $\mathrm{d} V / \mathrm{d} t_{\max }$ were not included. This is also the case for various studies where $I_{\mathrm{to1}}$ was blocked by drugs. The $I_{\mathrm{to1}}$ activator NS5806 decreased $\mathrm{d} V / \mathrm{d} t_{\max }$, suggesting that $I_{\text {to1 }}$ indeed modulates $\mathrm{d} V / \mathrm{d} t_{\max }$ [3]. However, NS5806 also reduced the fast sodium current $\left(I_{\mathrm{Na}}\right)$ [3], which is responsible for the rapid cardiac AP upstroke [4].

In the present study, we evaluated the effects of $I_{\mathrm{to}, \mathrm{f}}$ on depolarization and repolarization velocity as well as AP overshoot using the dynamic clamp technique [5]. All three characteristics were measured in HEK-293 cells transfected with $I_{\mathrm{Na}}$ channels to avoid interference of other depolarizing and repolarizing membrane currents.

\section{Methods}

\subsection{Patch-clamp experiments}

HEK-293 cells were transiently transfected with $0.3 \mu \mathrm{g}$ SCN5A cDNA and $0.3 \mu \mathrm{g} \beta 1$ cDNA (both wild-type), encoding the $\alpha$ - and $\beta$-subunits of the cardiac $I_{\mathrm{Na}}$ channel, respectively.

The alternating voltage clamp/current clamp (VC/CC) technique was used to measure $I_{\mathrm{Na}}$-driven upstrokes at physiological $\mathrm{Na}^{+}$concentrations and temperature $\left(37^{\circ} \mathrm{C}\right)$ in HEK-293 cells, as described previously [4]. In short, cells were voltage clamped at a holding potential of -85 $\mathrm{mV}$, similar to the resting membrane potential of ventricular myocytes, and upstrokes, overshoot, and early repolarization were elucidated by switching to the current clamp mode of the patch clamp technique for $20 \mathrm{~ms}$.

\subsection{Dynamic clamp}

$I_{\mathrm{to}, \mathrm{f}}$ of the Bondarenko et al. mouse ventricular AP model [6] was computed in real time and injected, in a 

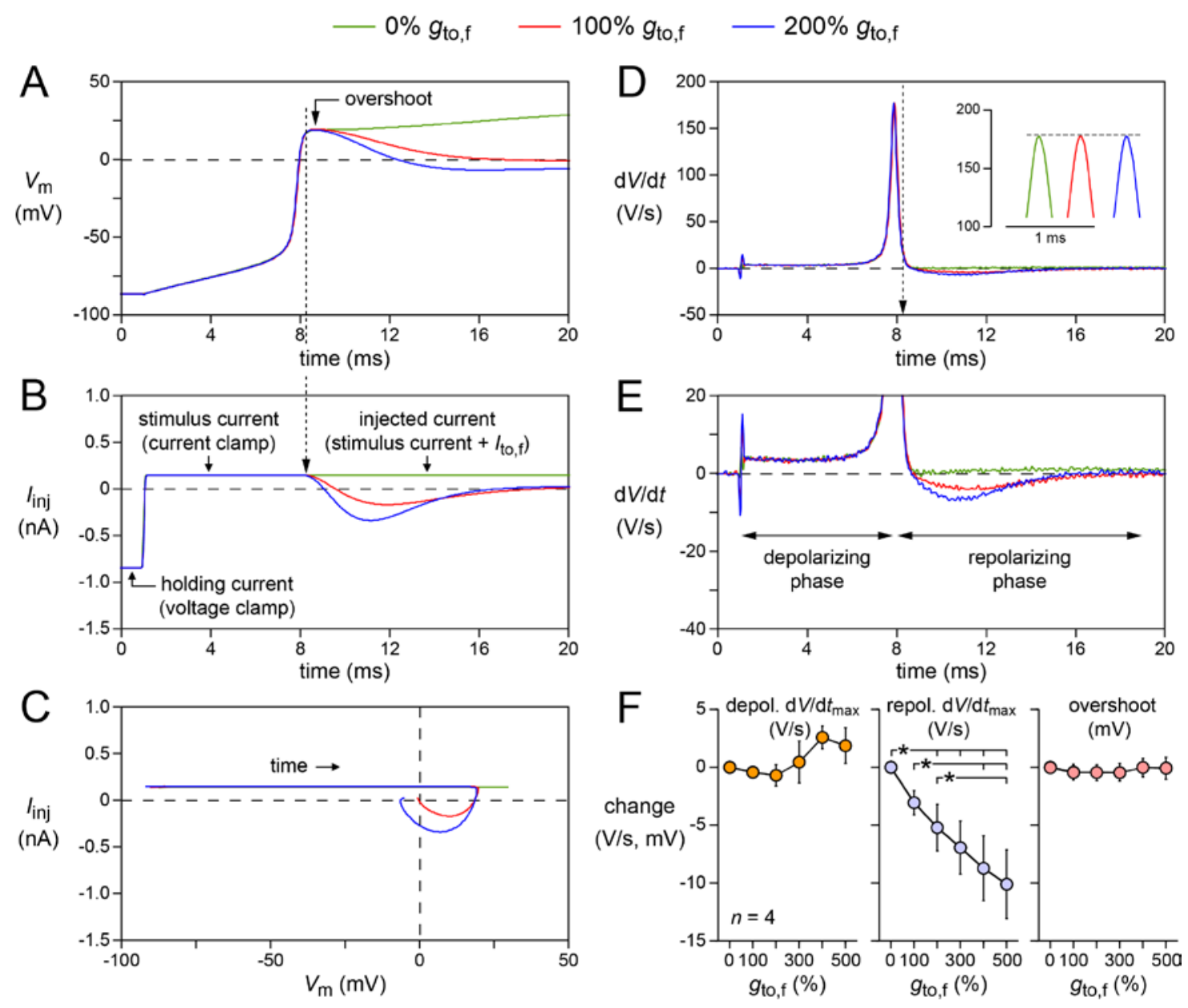

Figure 1. Effect of $I_{\mathrm{to}, \mathrm{f}}$ density $\left(g_{\mathrm{to}, \mathrm{f}}\right)$ on fast depolarization, repolarization, and overshoot. (A) Typical superimposed membrane potential recordings without $I_{\mathrm{to}, \mathrm{f}}\left({ }^{\prime} 0 \% g_{\mathrm{to}, \mathrm{f}}\right.$ '), with the default model $I_{\mathrm{to}, \mathrm{f}}$ settings (' $100 \% g_{\mathrm{to}, \mathrm{f}}$ '), and with doubled $I_{\mathrm{to}, \mathrm{f}}$ density (' $200 \% g_{\mathrm{to}, \mathrm{f}}$ '). (B) Injected current $\left(I_{\mathrm{inj}}\right)$, demonstrating activation of $I_{\mathrm{to}, \mathrm{f}}$, during the recordings of panel A. (C) Phase plane plot of $I_{\text {inj }}$ versus $V_{\mathrm{m}}$. (D) First derivative of the membrane potential recordings of panel A $(\mathrm{d} V / \mathrm{d} t)$. Inset: maximal $\mathrm{d} V / \mathrm{d} t$ during depolarization. (E) Effect of $I_{\mathrm{to}, \mathrm{f}}$ density on $\mathrm{d} V / \mathrm{d} t$ during repolarization. (F) Changes in depolarizing $\mathrm{d} V / \mathrm{d} t$, repolarizing $\mathrm{d} V / \mathrm{d} t$, and overshoot upon an increase in $g_{\mathrm{to}, \mathrm{f}}(n=4)$. ${ }^{*} P<0.05$.

continuous feedback loop, into the real cell during its upstroke. The $I_{\mathrm{to}, \mathrm{f}}$ reversal potential was set to $-86 \mathrm{mV}$, equal to the potassium equilibrium potential. $I_{\mathrm{to}, \mathrm{f}}$ density, activation rate and voltage dependence were varied.

\subsection{Statistics}

Data are presented as mean \pm SEM. One-way repeated measures ANOVA was used for comparing the effects of $I_{\mathrm{to}, \mathrm{f}}$ density, activation rate, and voltage dependence. $P<0.05$ was considered statistically significant.

\section{Results}

Fig. 1 shows the effect of $I_{\mathrm{to}, \mathrm{f}}$ density $\left(g_{\mathrm{to}, \mathrm{f}}\right)$ on depolarization and repolarization velocity and overshoot.
As illustrated in Fig. 1, A and D-F, Ito,f modulates repolarization velocity without affecting $\mathrm{d} V / \mathrm{d} t_{\max }$ of depolarization and overshoot. The effect on repolarization increases with increasing $g_{\mathrm{to}, \mathrm{f}}$ (Fig. 1F). The lack of effect on depolarization and overshoot is likely due to the relatively slow activation of $I_{\mathrm{t}, \mathrm{f}}$ and the resulting virtual absence of $I_{\mathrm{to}, \mathrm{f}}$ during these AP phases (Fig. 1, B and C).

The $I_{\mathrm{to}, \mathrm{f}}$ equations of Bondarenko et al. [6] are based on experimental data obtained at room temperature and the Bondarenko et al. AP model "is nominally adjusted for room temperature of $25^{\circ} \mathrm{C}$ " [6]. Fig. 2 shows the effect of an increase in $I_{\mathrm{to}, \mathrm{f}}$ activation rate, thereby representing a more close-to-physiological temperature, on depolarization and repolarization velocity and overshoot. As illustrated in Fig. 2, A and D-F, the increase in $I_{\mathrm{to}, \mathrm{f}}$ activation rate results in a minor, but significant, decrease in both $\mathrm{d} V / \mathrm{d} t_{\max }$ of depolarization 


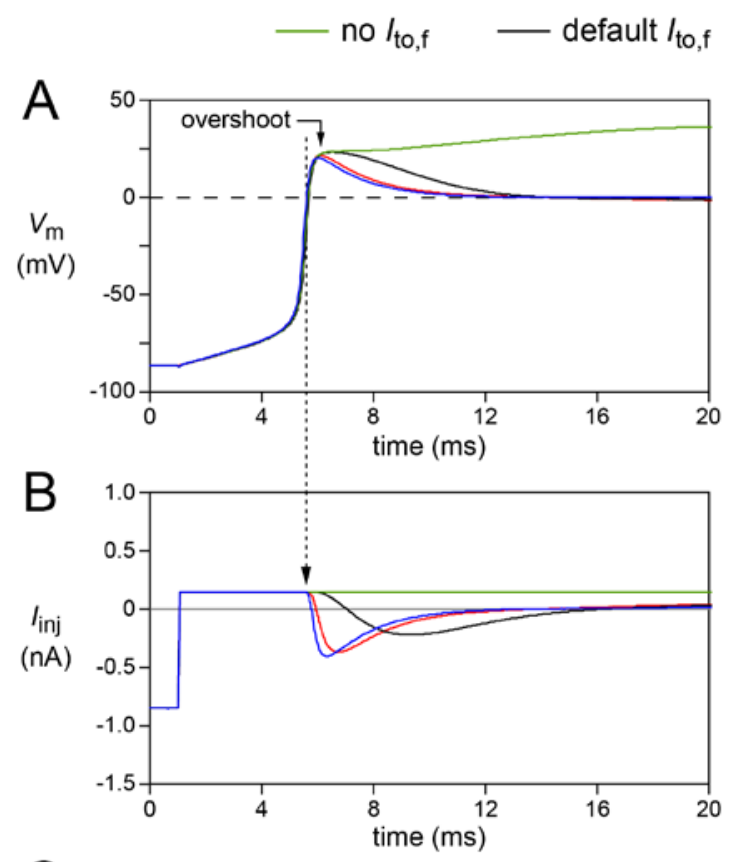

$-5 \times$ faster

$10 \times$ faster
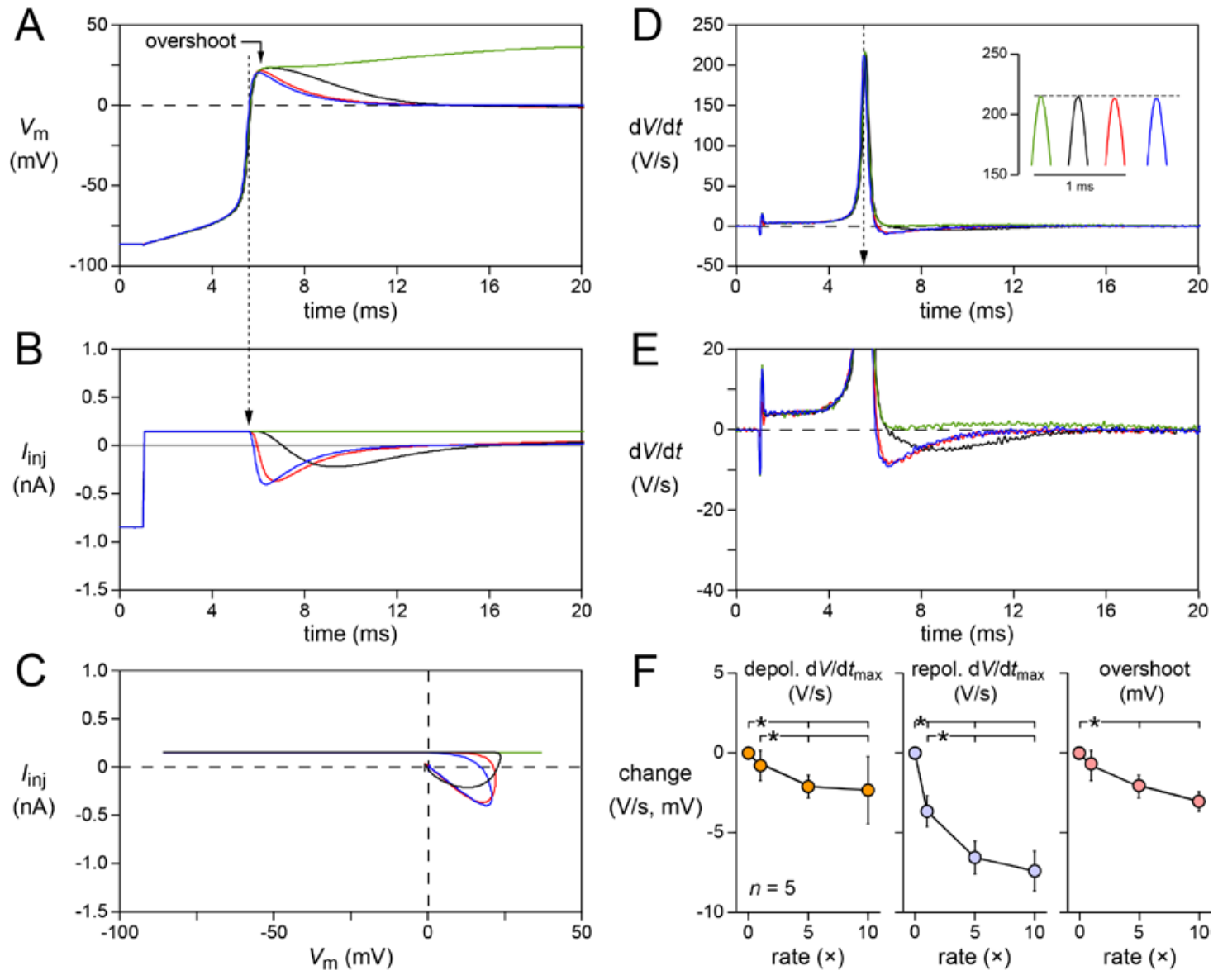

Figure 2. Effect of $I_{\mathrm{to}, \mathrm{f}}$ activation rate on fast depolarization, repolarization, and overshoot. (A) Typical superimposed membrane potential recordings without $I_{\mathrm{to}, \mathrm{f}}$ ('no $I_{\mathrm{to}, \mathrm{f}}$ '), with the default model $I_{\mathrm{to}, \mathrm{f}}$ activation rate ('default $I_{\mathrm{to}, \mathrm{f}}$ '), and with 5 and 10 times faster activation (' $5 \times$ faster' and ' $10 \times$ faster', respectively). (B) Injected current $\left(I_{\text {inj }}\right)$, demonstrating activation of $I_{\mathrm{to}, \mathrm{f}}$, during the recordings of panel A. (C) Phase plane plot of $I_{\mathrm{inj}}$ versus $V_{\mathrm{m}}$. (D) First derivative of the membrane potential recordings of panel A $(\mathrm{d} V / \mathrm{d} t)$. Inset: maximal $\mathrm{d} V / \mathrm{d} t$ during depolarization. (E) Effect of $I_{\mathrm{to}, \mathrm{f}}$ activation rate on $\mathrm{d} V / \mathrm{d} t$ during repolarization. (F) Changes in depolarizing $\mathrm{d} V / \mathrm{d} t$, repolarizing $\mathrm{d} V / \mathrm{d} t$, and overshoot upon an increase in $I_{\mathrm{to}, \mathrm{f}}$ activation rate $(n=5) . * P<0.05$.

and overshoot, likely because $I_{\mathrm{to}, \mathrm{f}}$ is now activated during these AP phases (Fig. 2, B and C).

Electrophysiological properties of $I_{\text {to1 }}$ are frequently measured in the presence of $\mathrm{Cd}^{2+}$ or $\mathrm{Co}^{2+}$ to block contaminating $\mathrm{Ca}^{2+}$ currents. Unfortunately, these divalent ions induce a positive shift in voltage dependence of $I_{\text {to1 }}$. Accordingly, Bondarenko et al. [6] have taken into account an $\approx 10 \mathrm{mV}$ shift in their $I_{\mathrm{to}, \mathrm{f}}$ equations. However, the shift in voltage dependence of $I_{\mathrm{to}, \mathrm{f}}$ is not exactly known. Therefore, we performed a final series of dynamic clamp experiments with a 5 times faster $I_{\mathrm{to}, \mathrm{f}}$ activation and shifts in $I_{\mathrm{to}, \mathrm{f}}$ voltage dependence ranging from 0 to -50 $\mathrm{mV}$ (Fig. 3). Negative shifts result in a decreased $\mathrm{d} V / \mathrm{d} t_{\max }$ of depolarization, faster repolarization, and a smaller overshoot. The effects are more pronounced with larger shifts (Fig. 3F).

\section{Conclusion}

We conclude that (1) $I_{\mathrm{to}, \mathrm{f}}$ clearly affects repolarization, and (2) $I_{\mathrm{to}, \mathrm{f}}$ may modulate $\mathrm{dV} / \mathrm{d} t_{\max }$ of depolarization and overshoot, but only if its activation is fast and its activation threshold is rather negative. The minor effect on upstroke velocity suggests a limited role of $I_{\mathrm{to}, \mathrm{f}}$ in AP depolarization and overshoot.

\section{References}

[1] Guo W, Li H, London B, Nerbonne JM. Functional consequences of elimination of $I_{\mathrm{to}, \mathrm{f}}$ and $I_{\mathrm{to}, \mathrm{s}}$ : early afterdepolarizations, atrioventricular block, and ventricular arrhythmias in mice lacking Kv1.4 and expressing a dominant-negative Kv4 $\alpha$ subunit. Circ Res 2000;87:73-9. 

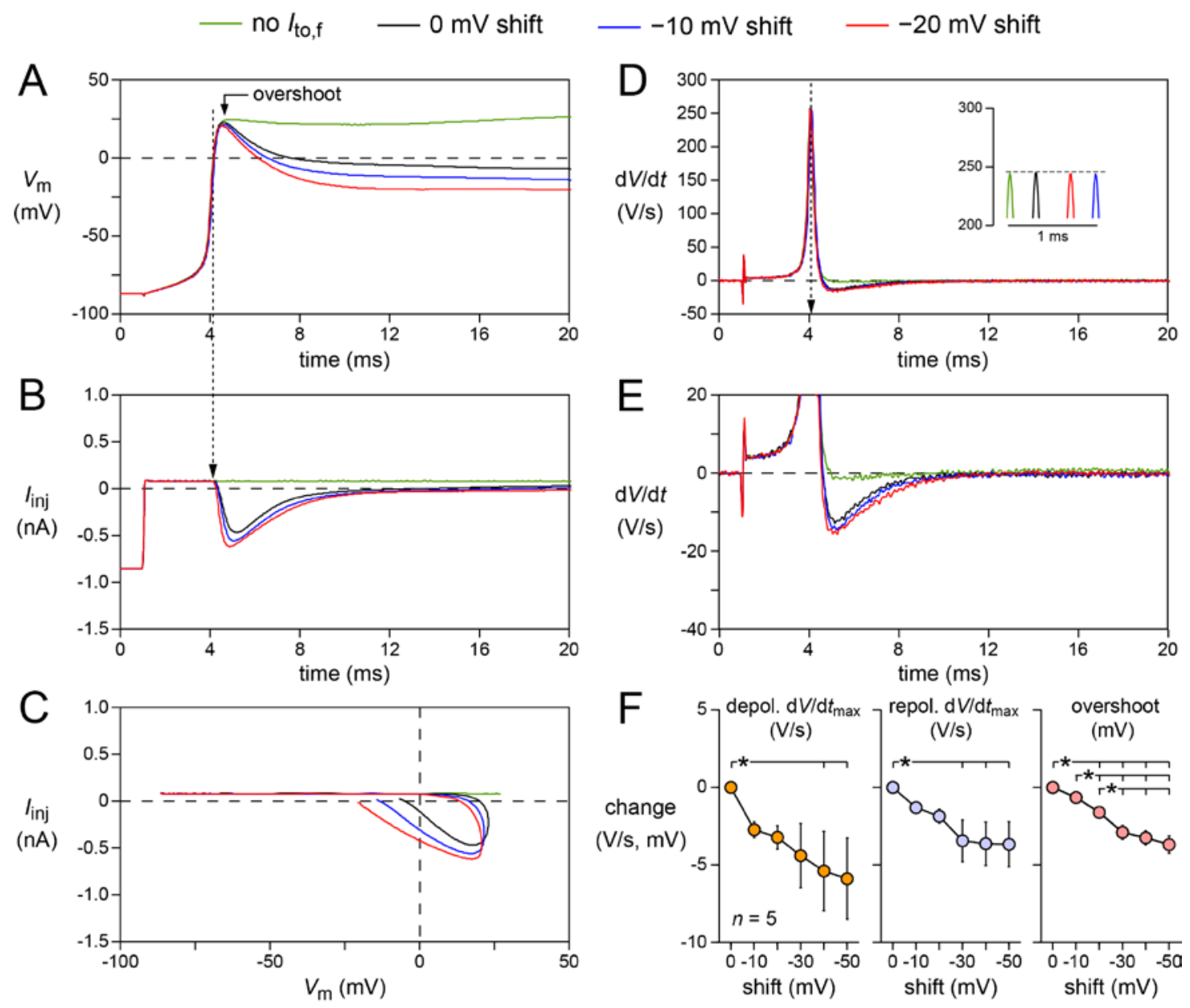

Figure 3. Effect of a negative shift in voltage dependence of $I_{\mathrm{to}, \mathrm{f}}$, in addition to a 5 times faster activation, on fast depolarization, repolarization, and overshoot. (A) Typical superimposed membrane potential recordings without $I_{\mathrm{to}, \mathrm{f}}$ ('no $I_{\mathrm{to}, \mathrm{f}}$ '), without a shift in voltage dependence (' $0 \mathrm{mV}$ shift'), and with a -10 or $-20 \mathrm{mV}$ shift in voltage dependence of $I_{\mathrm{to}, \mathrm{f}}$ ('-10 mV shift' and ' $-20 \mathrm{mV}$ shift', respectively). (B) Injected current $\left(I_{\mathrm{inj}}\right)$, demonstrating activation of $I_{\mathrm{to}, \mathrm{f}}$, during the recordings of panel A. (C) Phase plane plot of $I_{\text {inj }}$ versus $V_{\mathrm{m}}$. (D) First derivative of the membrane potential recordings of panel A (dV/dt). Inset: maximal $\mathrm{d} V / \mathrm{d} t$ during depolarization. (E) Effect of $I_{\mathrm{to}, \mathrm{f}}$ voltage dependence on $\mathrm{d} V / \mathrm{d} t$ during repolarization. (F) Changes in depolarizing $\mathrm{d} V / \mathrm{d} t$, repolarizing $\mathrm{d} V / \mathrm{d} t$, and overshoot upon a negative shift in voltage dependence of $I_{\mathrm{to}, \mathrm{f}}(n=5) .{ }^{*} P<0.05$.

[2] Liu J, Kim K-H, Morales MJ, Heximer SP, Hui C-C, Backx $\mathrm{PH}$. Kv4.3-encoded fast transient outward current is presented in Kv4.2 knockout mouse cardiomyocytes. PLoS One 2015;10: 0133274.

[3] Calloe K, Nof E, Jespersen T, Di Diego JM, Chlus N, Olesen S-P, Antzelevitch C, Cordeiro JM. Comparison of the effects of a transient outward potassium channel activator on currents recorded from atrial and ventricular cardiomyocytes. J Cardiovasc Electrophysiol 2011;22: 1057-66.

[4] Berecki G, Wilders R, de Jonge B, van Ginneken ACG, Verkerk AO. Re-evaluation of the action potential upstroke velocity as a measure of the $\mathrm{Na}^{+}$current in cardiac myocytes at physiological conditions. PLoS One 2010; 5:e15772.
[5] Wilders R. Dynamic clamp: a powerful tool in cardiac electrophysiology. J Physiol 2006;576:349-59.

[6] Bondarenko VE, Szigeti GP, Bett GCL, Kim S-J, Rasmusson RL. Computer model of action potential of mouse ventricular myocytes. Am J Physiol Heart Circ Physiol 2004;287:H1378-403.

Address for correspondence:

Arie O Verkerk, $\mathrm{PhD}$

Department of Anatomy, Embryology and Physiology Academic Medical Center, University of Amsterdam Meibergdreef 15, 1105 AZ Amsterdam, The Netherlands Phone: +31-20-5664644, E-mail: a.o.verkerk@amc.uva.nl 